\title{
EXPONENTIAL STABILITY FOR A STRUCTURE WITH INTERFACIAL SLIP AND MEMORY
}

\author{
CARLOS A. RAPOSO ${ }^{\dagger}$, DAVID A. Z. VILLANUEVA, SANTOS D. M. BORJAS AND DUCIVAL \\ C. PEREIRA
}

\author{
Date of Receiving : $\quad$ : 29.07.2016 \\ Date of Revision : $\quad 24.08 .2016$ \\ Date of Acceptance : 24.08 .2016
}

\begin{abstract}
In this work we consider a structure given by a laminated beam consisting of two identical layers uniform of length 1 , taking into account that an adhesive of the small thickness is bonding the two layers and produce the interfacial slip.

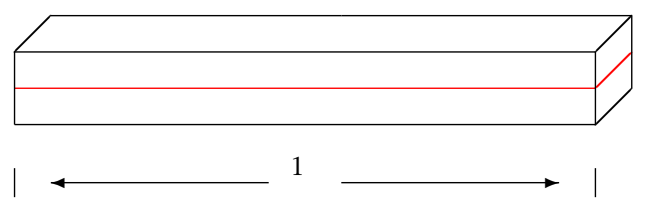

The memory effect with a dissipative relaxation function together with other stronger dissipative effects, for example the frictional damping, can not produce any rate of decay if the relaxation function does not decay uniformly. In this work we prove that the effect of the memory together with the frictional damping produces stabilization for this structure. We applied the Energy Method, that consists in the use of appropriated multiplies to build a functional of Lyapunov for the system.
\end{abstract}

\section{Introduction}

There are few papers that deal with systems of interfacial slip, we cite [1], [2], [3], [5], [6], [7] and references therein. We mention Hansen [3], Hansen and Spies [2] where the mathematical model (1.1)-(1.3) for two-layered beams with structural damping due to the interfacial slip was derived.

$$
\begin{array}{r}
\rho u_{t t}+G\left(\psi-u_{x}\right)_{x}=0, x \in(0,1), t \geq 0, \\
I_{\rho}\left(3 S_{t t}-\psi_{t t}\right)-G\left(\psi-u_{x}\right)-D\left(3 S_{x x}-\psi_{x x}\right)=0, x \in(0,1), t \geq 0, \\
3 I_{\rho} S_{t t}+3 G\left(\psi-u_{x}\right)+4 \delta_{0} S+4 \gamma_{0} S_{t}-3 D S_{x x}=0, x \in(0,1), t \geq 0 .
\end{array}
$$

2010 Mathematics Subject Classification. 93C20, 74D05, 35B40.

Key words and phrases. Exponential stability, laminated beam, interfacial slip, energy method, memory.

Communicated by. Octavio Paulo Vera Villagran

${ }^{\dagger}$ Corresponding author. 